\title{
Intracranial abscess after anterior skull base defect: does pneumocephalus play a role?*
}

\author{
Samuel S. Becker and Paul T. Russell \\ ${ }^{1}$ Department of Otolaryngology \& Head and Neck Surgery, Vanderbilt University Medical Center, \\ Nashville, TN, USA
}

SUMMARY

\begin{abstract}
Background: Intracranial complications of skull base defects are uncommon; however, they can be devastating when they do occur. Often these complications arise suddenly and without warning. Identifying patients at risk for intracranial abscesses may help with early diagnosis and allow for early interventions.

Objective: We report on 2 patients with traumatic pneumocephalus who developed intracranial abscesses. Our objective was to evaluate these cases and investigate the relationship between the presence of pneumocephalus and the formation of intracranial infection.

Methods: Data was reviewed on the cases of 2 patients who developed intracranial abscesses after pneumocephalus in the context of skull base defects.

Results: Two patients presented to our institution separately with the presence of intracranial abscesses. Both patients had violation of their anterior skull base - one from endoscopic sinus surgery, one from blunt trauma. Both patients had massive pneumocephalus prior to their abscess formation.

Conclusions: Intracranial abscess formation, traumatic skull base defects, and massive pneumocephalus are uncommon entities, which may be associated. It may be prudent to have heightened suspicion for the presence of intracranial abscess formation in patients with massive pneumocephalus secondary to anterior skull base trauma.
\end{abstract}

Key words: intracranial, abscess, pneumocephalus, sinusitis, anterior, skull base, defect

\section{INTRODUCTION}

Bony defects of the anterior skull base may arise from several sources. Blunt force trauma and iatrogenic, surgical trauma may acutely lead to dehiscences in the skull base with associated cerebrospinal fluid (CSF) leaks or - less commonly - pneumocephalus. Chronic, erosive processes such as sinonasal tumors, meningo-encephelocoeles, allergic fungal sinusitis, and mucocoeles may also result in anterior skull base defects. Treatments vary, but almost always involve surgical repair of the skull base defect.

Defects of the anterior skull base may lead to serious complications. The broken barrier between the sinonasal and intracranial cavities may present with CSF leaking out through the nose, or with air entering into the previously airtight cranium (pneumocephalus). These CSF leaks occur in roughly 3\% of all closed head injuries ${ }^{(1)}$. The incidence of iatrogenic CSF leaks from surgical trauma is thought to be less than $1 \%{ }^{(2)}$. It has, however, been suggested, that occult CSF leaks may occur in up to $3 \%$ of cases although this has not been confirmed ${ }^{(3,4)}$. In one recent study, $25 \%$ of otolaryngologists who responded to a questionnaire reported causing at least $1 \mathrm{CSF}$ leak during the previous 5 years ${ }^{(5)}$.

Pneumocephalus is most common after blunt trauma to the anterior skull base, and may occur in up to $30 \%$ of patients who suffer skull base trauma ${ }^{(6)}$. Patients with pneumocephalus have been noted to have a $25 \%$ accompanying risk of meningitis ${ }^{(7)}$. It has, furthermore, been postulated that there may be a close relationship between the presence of a CSF leak and pneumocephalus in that the egress of CSF creates a pressure gradient favorable for the ingress of air into the cranial cavity (8).

Diagnosis of CSF leaks begins with a high degree of suspicion particularly in any case of unilateral, clear rhinorrhea. While most CSF leaks are associated with accidental or surgical trauma ${ }^{(9)}$, tumors, congenital skull base malformations, and benign intracranial hypertension may also present with CSF leaks. Once suspicion is present, definitive diagnosis relies on appropriate laboratory testing. Testing for beta- 2 transferrin - a protein found only in CSF, perilymph, and aqueous humor - is 
both highly sensitive and specific, and requires less than $0.5 \mathrm{cc}$ of nasal fluid for testing ${ }^{(10,11)}$. More recently, an alternative test - for beta-trace protein - has been found to be similarly reliable for the diagnosis of CSF leaks ${ }^{(12-14)}$. These tests will confirm the presence of a CSF fistula, and rule out more benign sources of rhinorrhea such as vasomotor rhinitis.

Diagnosis of pneumocephalus is made with radiological imaging - most commonly computed tomography (CT). In most situations, small air bubbles are seen intrancranially. On occasion - particularly in cases of tension pneumocephalus - large volumes of air can be seen along with an associated mass effect on the intracranial contents. Patients with tension pneumocephalus may present with headache, altered mental status, and focal neurological findings. While cases of tension pneumocephalus after endoscopic sinus surgery have been documented, they are very rare ${ }^{(15)}$. In a review of 6,801 sinus procedures May et al. reported not a single case of pneumocephalus (16).

Left untreated, skull base defects with their associated CSF leaks and pneumocephalus may set the stage for such complications as meningitis, intracranial abscesses, and dural sinus thrombosis. Meningitis occurs in up to $25 \%$ of untreated CSF leaks, and in $10 \%$ of post-traumatic patients within 1 week of injury ${ }^{(17,18)}$. While the majority of traumatic CSF leaks resolve spontaneously, up to $10 \%$ of these patients will develop meningitis despite the apparent resolution of their fistula. There is strong data to support the surgical repair of any CSF leak ${ }^{(19)}$ as a means to prevent meningitis. Moreover, as has also been recently documented, greater than $20 \%$ of patients with untreated skull base defects present with life-threatening intracranial complications including meningitis and brain abscesses ${ }^{(20)}$.

As noted above, pneumocephalus is a common occurrence after blunt force or iatrogenic surgical trauma to the skull base. Once the barrier between the air filled sinonasal cavity, and the cranium is violated, air may flow into the cranial cavity. In most instances, however, this retrograde flow is minimal due to the resistance provided by the intra-cranial contents. Resolution of pneumocephalus is spontaneous in the majority of cases. On occasion, however, massive pneumocephalus may occur and - when it does - usually occurs in the context of tension pneumocephalus. Tension pneumocephalus may occur via a "ball-valve" effect in which anatomical distortion lends itself to air entry into the intracranial cavity without any means of efflux. An alternative theory is of an "inverted bottle" mechanism by which - with the leakage of cerebrospinal fluid - a negative pressure is created through which air is drawn into the cavity ${ }^{(21)}$.

A small number of skull base defects may be complicated by intracranial abscesses. Patients whose skull base defects are secondary to endoscopic sinus surgery may have an elevated risk for abscess, since they - presumably - have bacterial overgrowth in their sinonasal cavity as an indication of their initial sinus procedure ${ }^{(22)}$. An iatrogenic skull base defect in this context places a focus of contamination in contiguity with the cranial cavity ${ }^{(23)}$. More common, however, are intracranial abscess that arise after violation of the anterior skull base by an infected mucocoele caused by blocked sinus drainage from a fibro-osseus lesion ${ }^{(24-26)}$. In some cases, the mucocoele may arise without the presence of an associated lesion, and may erode on its own through the skull base setting up a nidus for intracranial infection ${ }^{(27)}$.

Of the 10 percent of brain abscesses that arise secondary to direct seeding from trauma or neurosurgical procedure, bacterial sources are variable and include aerobic bacteria such as Streptococcus milleri, Streptococcus viridans, Staphylococcus aureus, and beta-hemolytic streptococci, and anaerobic bacteria such as Bacteroides species. Diagnosis is dependent on a high degree of suspicion. Headache, fever, vomiting, altered mental status, seizure, hemiparesis, and cranial nerve signs are concerning for an intracranial process and should be rigorously investigated ${ }^{(28)}$. While MRI has been found to have greatest accuracy in the diagnosis of intracranial processes ${ }^{(29,30)}$, CT may also play a role in the identification of skull base defects that allow cross-contamination of the sterile brain cavity from the non-sterile nose and sinuses ${ }^{(31)}$.

As discussed above, once the barrier between the sterile cranial cavity and the non-sterile sinonasal cavity is breached, meningitis, intra-cranial abscess, and dural sinus thrombosis are of real concern. In most cases, however, repair of the defect and re-establishment of a barrier prevent these worrisome complications. Treatment, as with any abscess, is primarily surgical followed by broad-spectrum antibiotics. Steroid and anti-seizure medications are also used in most instances ${ }^{(32)}$. Surgery may be performed by an otolaryngologist, a neurosurgeon, or a joint approach involving a neurosurgeon and an otolaryngologist. The specifics of the repair will vary depending on the situation. It may be possible to narrow the antibiotic spectrum based on culture results, however, these patients have often been on a multitude of antibiotics limiting the utility of cultures.

Skull base defect repair may be performed via external, endoscopic endonasal, or a combined approach. In the case of an uncomplicated CSF leak, most repairs are performed via endoscopic endonasal approach. Repair materials vary and include autologous tissue (free mucosal graft, septal cartilage, septal bone, calvarial bone, abdominal fat), allogenic tissue (cadaveric iliac crest, Alloderm), and artificial materials and glues ${ }^{(33-35)}$. While defects larger than $1 \mathrm{~cm}$ have traditionally involved the use of a bone or cartilage graft to support the cranium and prevent a secondary encephalocele (36), many have reported suc- 
cess with soft tissue grafts only in the repair of large skull base defects ${ }^{(37)}$. In some cases of particularly large defects, surgeons have opted for the use vascularized local flaps and vascularized free tissue flaps for skull base repair ${ }^{(38,39)}$. The use of lumbar subarachnoid drains in similarly controversial and high success rates have been reported both with and without their use ${ }^{(40)}$. Repair of uncomplicated pneumocephalus is traditionally performed in the same manner as for CSF leaks.

Tension pneumocephalus is a life-threatening emergency that requires immediate treatment. Emergent decompression is performed via neurosurgical techniques - most commonly burr hole placement and ventriculostomy. Once the emergency situation has resolved, curative treatment involves closure of the skull base defect. Lumbar drains should be avoided in these cases, as they may create a negative pressure gradient that favors intracranial flow of air from the sinonasal cavity. Definitive repair has traditionally been via neurosurgical craniotomy approach. Over the past decade, however, endoscopic endonasal approaches have increasingly been employed ${ }^{(41)}$.

With proper management, complications of skull base defects are rare - but may include CSF leaks, pneumocephalus, meningitis, intracranial abscesses, and dural sinus thrombosis. The purpose of this study is to review 2 cases of intracranial abscesses after repair of skull base defects in an attempt to identify any contributing factors to abscess formation. We also conduct a review of the literature on this topic.

\section{METHODS}

After approval by the Human Investigation Committee, chart review was undertaken of 2 patients recently treated at the Vanderbilt University Medical Center (VUMC) Department of Otolaryngology-Head and Neck Surgery. These 2 patients were notable for having developed intracranial abscesses shortly after endoscopic endonasal repair of anterior skull base defects with associated tension pneumocephalus.

\section{RESULTS}

\section{Case 1}

Patient 1 is an otherwise healthy 9 year old boy who suffered multiple injuries during a motor vehicle accident including a closed head injury, bilateral frontal lobe contusions, subarachnoid hemorrhage, splenic inury, pneumothorax, and femur, ankle, and wrist fractures. Facial fractures include bilateral comminuted displaced naso-orbital ethmoid fractures, and bilateral Le Fort I and Le Fort II fractures. On Post-trauma day 1 the patient was taken to the operating room by the neurosurgical service where the depressed frontal bone fracture was elevated and repaired. Mid-face fractures were repaired by the Plastic Surgery service. The patient had a favorable recovery and was discharged on post-trauma day 30 . He returned for routine follow up in the neurosurgery clinic on post-trauma day 49 complaining of recent onset of headaches and unilateral clear rhin-

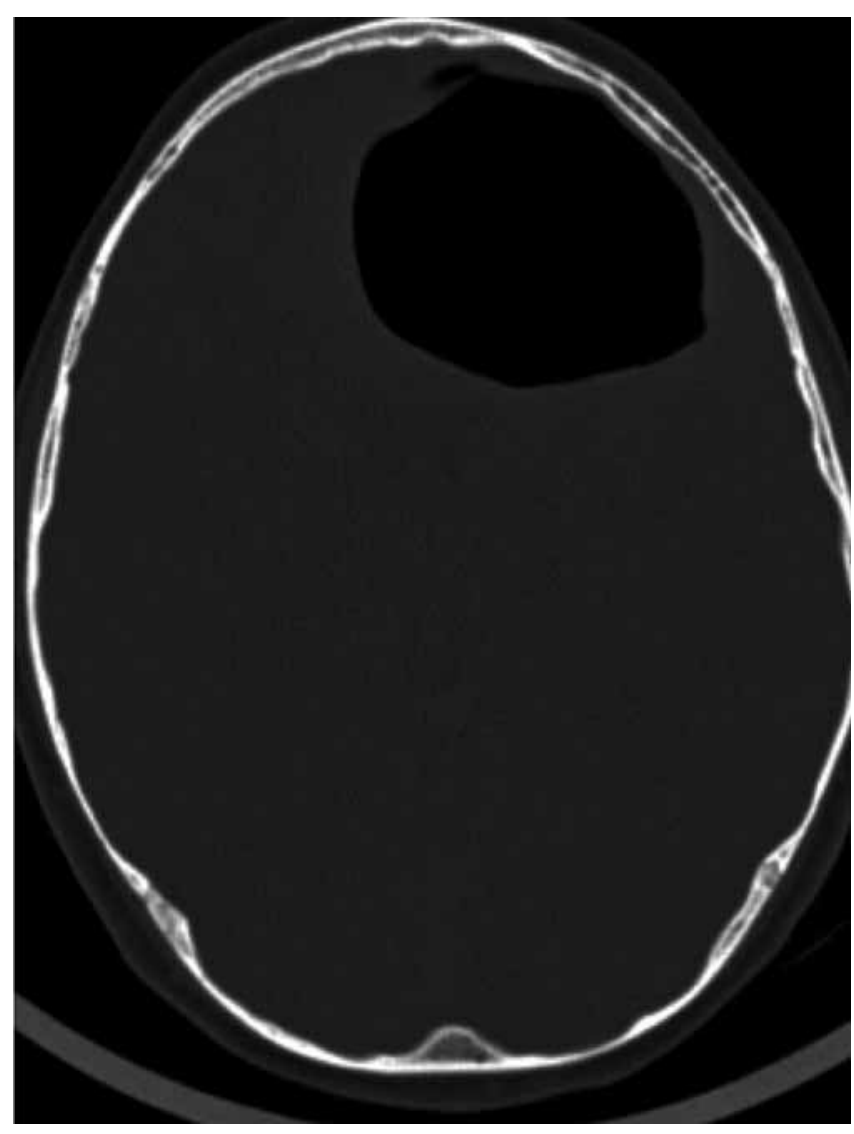

Figure 1. This axial CT scan demonstrates the presence of significant intracranial air (pneumocephalus).

orrhea. Computed tomography (CT) scan demonstrated massive left-sided pneumocephalus (Figure 1). The patient was taken the following day to the operating room by the VUMC Otolaryngology service where endoscopic repair of a $10 \times 15$ $\mathrm{mm}$ anterior skull base defect was performed using standard underlay and overlay techniques. The patient was then discharged on post-operative day 5 after an uneventful hospital stay. He then represented to the Emergency room 51 days after the endoscopic repair of his skull base defect with never, nausea, and vomiting. CT scan revealed a $6 \mathrm{~cm}$ left-sided frontal lobe abscess (Figure 2). Neurosurgical drainage via endoscopic aspiration produced approximately $100 \mathrm{cc}$ of purulence which grew Methicillin resistant staphylococcus aureus (MRSA) in culture. Treatment consisted of a 12 week course of vancomycin, rifampin, and metronidazole. At 12 week follow-up, the patient had made a significant clinical recovery, and CT demonstrated near-resolution of the abscess (Figure 3).

\section{Case 2}

Patient 2 is 46 year old woman referred to VUMC 15 days after septoplasty and functional endoscopic sinus surgery complicated by intra-operative violation of the anterior skull base and resultant cerebrospinal fluid (CSF) leak. The skull base penetration was recognized at the time of surgery, and repair was undertaken by the otolaryngologist with neurosurgical assistance and placement of a lumbar subarachnoid drain. The 


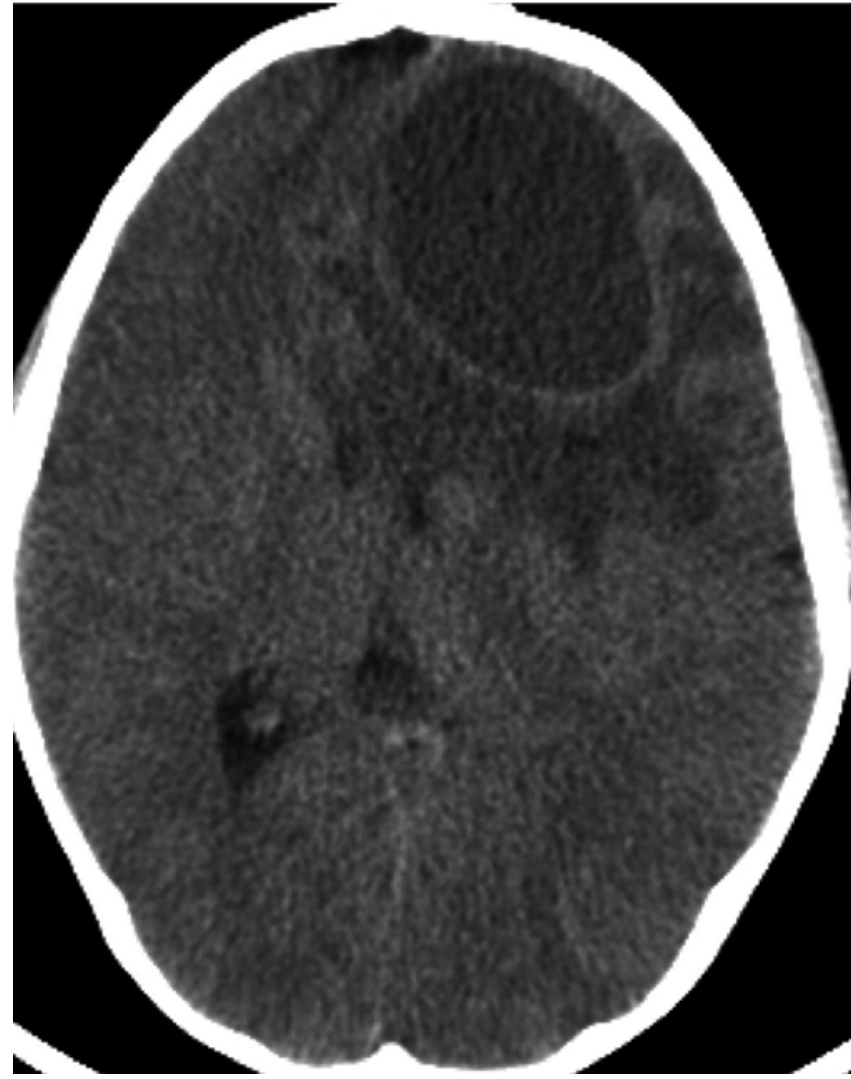

Figure 2. In this axial CT (soft tissue windowing) taken 51 days after the image in Figure 1, the patient has developed a ring-enhancing abscess at the site of the former pneumocephalus.

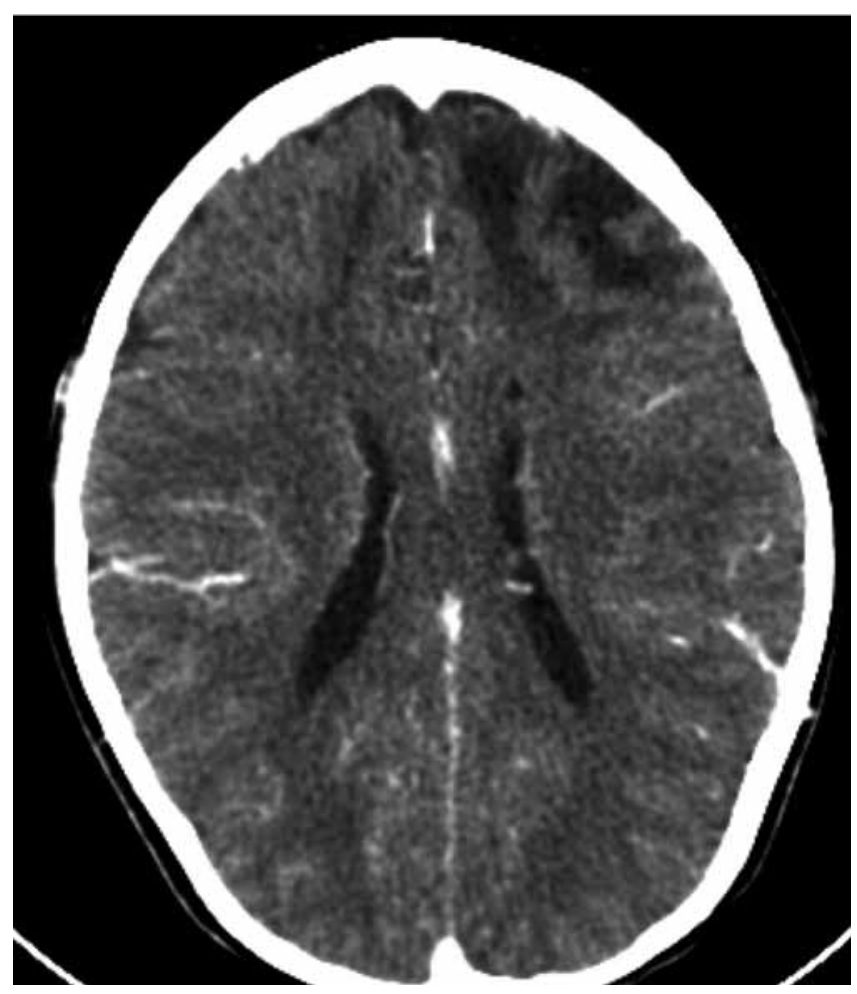

Figure 3. After surgical and medical management, the abscess seen in Figure 2 has almost completely resolved. There is slight residual edema apparent on this axial $\mathrm{CT}$ image.

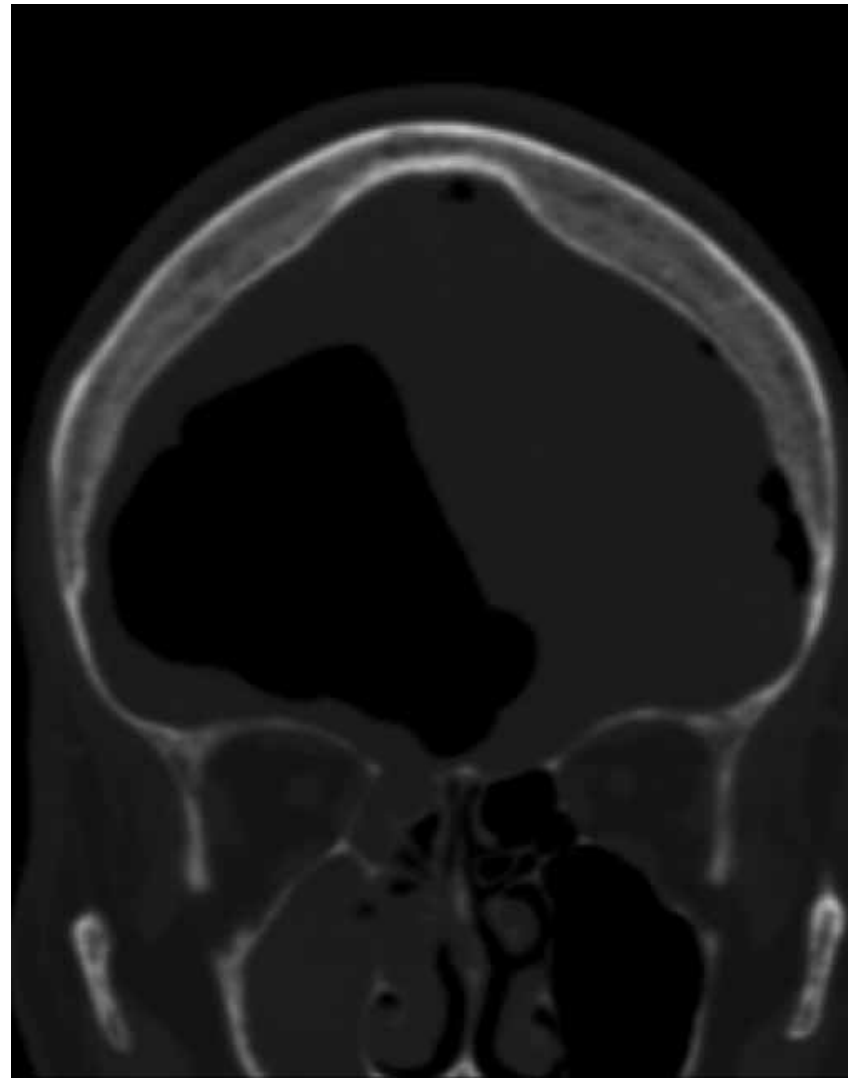

Figure 4. A right sided skull base defect and massive pneumocephalus are apparent on on this coronal $\mathrm{CT}$ image.

patient was then discharged after an uneventful 7 day hospital stay, only to return several days later with unilateral rhinorrhea and headaches. CT demonstrated massive right-sided (tension) pneumocephalus (Figure 4). Surgical repair at VUMC of a $12 \mathrm{x}$ $25 \mathrm{~mm}$ skull base defect was performed via standard underlay and overlay techniques. As in Case 1, carved cadaveric iliac crest bone was used as the primary support for the underlay graft. The patient was then discharged on post-operative day 3 after an uneventful hospital stay. 4 days later the patient returned to clinic doing well; CT demonstrated resolving pneumocephalus. The patient then presented to the VUMC emergency room with fever, chills, night sweats, and headache 68 days after her skull base repair at Vanderbilt. MRI demonstrated a $3 \mathrm{~cm}$ right frontal lobe abscess. Neurosurgical drainage of gross purulence was performed via an open approach during which the previously placed skull base repair was removed and a pericranial flap was laid down. Although cultures from the abscess demonstrated no growth, treatment was continued with 8 weeks of Vancomycin, Cefepime, and Metronidazole. The patient was discharged 5 days after her abscess drainage, but returned 11 days later with CSF leakage. She was taken back to the operating room by the neurosurgical service for revision of her pericranial flap. At 2 month follow up after this revision, she was clinically well with no signs or symptoms of CSF leak or intracranial infection. MRI showed continued resolution of her abscess. 


\section{DISCUSSION}

When an intracranial abscess occurs in association with a skull base defect, neurosurgical involvement is imperative and often involves otolaryngologists for defects that are favorably located for an endoscopic approach. Abscess drainage will most commonly involve an external approach - usually craniotomy. In some cases, as in Case 1 described in this paper, drainage may be via an externally based endoscopic approach. As with nontension pneumocephalus, lumbar drains should be avoided.

Both patients in our study suffered the formation of intracranial abscesses months after the occurrence of skull base defects, one from blunt trauma, the other from surgical trauma. Management of skull base defects is common at our institution. In a recent review (unpublished data) of 41 endoscopic endonasal repairs of skull base defects, there were no major complications other than these 2 described. These 2 patients stand out both for their abscess formation several months after their initial trauma, as well as for the presence of pneumocephalus. It is in this context that we suggest that these 2 processes may be related.

Massive sustained pneumocephalus - as was present in both of these patients - leads to compression of the intracranial contents within the rigid cranium. As with any organ, sustained compression leads to ischemia, and ischemia leads to micronecrosis which is a favorable environment for development of an intracranial abscess. Moreover, in these patients with massive pneumocephalus and violation of the anterior skull base, the sterile brain cavity has been contaminated by the non-sterile sinuses. An ischemic, traumatized contaminated environment whose primary defenses (the barrier between the sterile brain and the non-sterile sinuses) have been compromised may be an ideal situation for slow abscess formation.

Our findings suggest that it may be prudent to closely examine patients with massive pneumocephalus for any signs or symptoms of intracranial infection. In addition to the standard sinus CT, contrasted MRI or CT imaging may be of use in the post operative evaluation of these patients." These observations, however, are not conclusive, and warrant further investigation. An animal model is planned to investigate the role of massive pneumocephalus on intracranial abscess formation. In the meantime, until further data can be collected, we would suggest that patients with massive pneumocephalus in the context of skull base defects be followed with heightened suspicion for formation of intracranial processes.

\section{CONCLUSION}

Intracranial abscess formation, traumatic skull base defects, and massive pneumocephalus are uncommon entities which may be associated. It may be prudent to have heightened suspicion for the presence of intracranial abscess formation in patients with massive pneumocephalus secondary to anterior skull base trauma. MRI, or contrasted CT imaging may be of use in the evaluation of these patients.

\section{ACKNOWLEDGEMENTS}

There is nothing to declare and no conflict of interest.

\section{REFERENCES}

1. Mincy J. Post-traumatic spinal fluid fistulas of the frontal fossa. J Trauma 1966; 6: 618-622.

2. May M, Levine H, Mester S,et al. Complications of endoscopic sinus surgery: analysis of 2018 patients - incidence and prevention. Laryngoscope 1994; 104: 1080-1083.

3. Bachmann G, Djenabi U, Jungehülsing $M$, Petereit $H$, Michel $O$. Incidence of occult cerebrospinal fluid fistula during paranasal sinus surgery. Arch Otolaryngol Head Neck Surg. 2002; 128: 12991302.

4. Bachmann-Harildstad G. Incidence of CSF fistula after paranasal sinus surgery: the Northern Norwegian experience. Rhinology 2007; 45: 305-307.

5. Platt M, Shaye D, Parnes S. CSF fistula and ESS:Survey of 599 otolaryngologists in NY. Otolaryngol Head Neck Surg 2006; 135 (2 S1): 44.

6. Ommaya AK. Cerebrospinal fluid fistula and pneumocephalus. In: Wilkins RH, Rengachary SS, eds. Neurosurgery. 2nd ed. New York: McGraw-Hill; 1996: 2773-2782.

7. Noth JW. On the importance of intracranial air. Br J Surg 1971; 58 : 826-829.

8. Walker FO, Vern BA. The mechanism of pneumocephalus formation in patients with CSF fistulas. J Neuro Neuros Psych 1986; 49: 203-205.

9. Zlab MK, Moore GF, Daly DT, et al. Cerebrospinal fluid rhinorrhea: a review of the literature. Ear Nose Throat J 1992; 71: 314317.

10. Papadea C, Schlosser R. Sensitive detection of beta- 2 transferrin in cerebrospinal fluid leakage using an automated immunofixation electrophoresis method. Clin Chem 2004; 51: 464-470.

11. Warnecke A, Averbeck T, Wurster U, et al. Diagnostic relevance of Beta-2 transferrin for the detection of cerebrospinal fluid distulas. Arch Otolaryngol Head Neck Surg 2004; 130: 1178-1194.

12. G. Bachmann-Harildstad. Diagnostic values of beta-2 transferrin and beta-trace protein as markers for cerebrospinal fluid fistula. Rhinology 2008; 46: 82-85.

13. Arrer E, Meco C, Oberascher, et al. Beta-Trace Protein as a Marker for Cerebrospinal Fluid Rhinorrhea. Clin Chem 2002; 48: 939-941.

14. Meco C, Oberascher G, Arrer E et al. Beta-trace protein test: new guidelines for the reliable diagnosis of cerebrospinal fluid fistula. Otolaryngol Head Neck Surg 2003; 129: 508-517.

15. Campanelli J, Odland R. Management of tension pneumocephalus caused by endoscopic sinus surgery. Otolaryngol Head Neck Surg 1997; 116: 247-250.

16. May M. Levine H, Mester S, et al. Complications of endoscopic sinus surgery: analysis of 2018 patients - incidence and prevention. Laryngoscope 1994; 104: 1080-1083.

17. Daudia A, Biswas D, Jones N. Risk of meningitis with cerebrospinal fluid rhinorrhea. Ann Otol Rhinol Laryngol 2007; 116: 902-905.

18. Bernal-Sprekelsen M, Bleda-Vázquez C, Carrau RL. Ascending meningitis secondary to traumatic cerebrospinal fluid leaks. Am J Rhinol. 2000; 14: 257-259.

19. Bernal-Sprekelsen M, Alobid I, Mullol J, et al. Closure of cerebrospinal fluid leaks prevents ascending meningitis. Rhinology. 2005; 43: 277-281.

20. Harvey RJ, Smith JE, Wise SK, et al. Intracranial complications before and after endoscopic skull base reconstruction. Am J Rhinol. 2008; 22: 516-521. 
21. Markham JW. Pneumocephalus. In: Vinken PJ, Bruyn GD, eds. Hnadbook of clinical neurology. Vol 24. Amsterdam: North Holland Publishing Co., 1976: 201-213.

22. Kocaeli H, Hakyemez B, Bekar A, et al. Unusual complications and presentations of intracranial abscess: experience of a single institution. Surgical Neurology 2008; 69: 383-391.

23. Blomstedt G. Infections in neurosurgery: a retrospective study of 1143 patients and 1517 operations. Acta Neurochir 1985; 78: 81-90.

24. Koyuncu M, Belet U, Sesen T, et al. Huge osteoma of the frontoethmoidal sius with secondary brain abscess. Auris Nasus Larynx 2000; 27: 285-287.

25. Summers LE, Mascott CR, Tompkins JR, et al. Frontal sinus osteoma associated with cerebral abscess formation: a case report. Surg Neurol. 2001; 55: 235-239.

26. Aygun D, Sahin H. fibrous dysplasia of the frontal sinus: an uncommon cause of frontal lobe abscess. J Clin Neuroscience. 2004; 11: 904-906.

27. Malard O, Gayet-Delacroix M, Jegoux F, et al. Spontaneous sphenoid sinus mucocele revealed by meningitis and brain abscess in a 12-year-old child. Am J Neuroradiol. 2004; 28: 873-875.

28. Jones NS, Walker JL, Bassi S, Jones T, Punt J. The intracranial complications of rhinosinusitis: Can they be prevented? Laryngoscope. 2002; 112: 59-63.

29. Younis RT, Anand V, Davidson B. The role of computed tomography and magnetic resonance imaging in patients with sinusitis with complications. Laryngoscope. 2002: 112: 224-229.

30. Schroth G, Kretzschmar K, Gawehn J, et al. Advantage of magnetic resonance imaging in the diagnosis of cerebral infectins. Neuroradiology. 1987; 29: 120-126.

31. Yujin W, Babgsen S, Liming J, et al. CT diagnosis of 60 cases of small cerebral abscess. J Neuroradiol. 1988; 15: 77-84.

32. Younis RT, Lazar RH, Anand VK. Intracranial complications of sinusitis: a 15 year review of 39 cases. Ear Nose Throat J. 2002; 81: 637-644.

33. Schlosser RJ, Bolger WE. Endoscopic management of cerebrospinal fluid rhinorrhea. Otolaryngol Clin N America. 2006; 39: 523-538.

34. McMains KC, Gross CW, Kountakis SE. Endoscopic management of cerebrospinal fluid rhinorrhea. Laryngoscope. 2004; 114: 18331837.
35. Wormald PJ, McDonogh M. The bath-plug closure of anterior skull base cerebrospinal fluid leaks. Am J Rhinol. 2003; 17: 299 305.

36. Lorenz RR, Dean RL, Hurley DB, et al. Endoscopic reconstruction of anterior and middle cranial fossa defectsusing acellular dermal allograft. Laryngoscope. 2003; 113: 496-501.

37. Germani RM, Vivero R, Herzallah IR, et al. Endoscopic reconstruction of large anterior skull base defects using acellular dermal allograft. Am J Rhinol. 2007; 21: 615-618.

38. Pinheiro-Neto, Diogenes C, Prevedello D. Improving the design of he pedicled nasoseptal flap for skull base reconstruction: a radioanatomic study. Laryngoscope. 2007; 117: 1560-1569.

39. Weber SM, Kim JH, Wax MK. Role of free tissue transfer in skull base reconstruction. Otolaryngol Head Neck Surg. 2007; 136: $914-$ 919.

40. Casiano RR, Jassir D. Endoscopic cerebrospinal fluid rhinorrhea repair: is a lumbar drain necessary? Otolaryngol Head Neck Surg 1999; 121: 745-750.

41. Aferzon M, Aferzon J, Spektor Z. Endoscopic repair of tension pneumocephalus. Otolaryngol Head Neck Surg. 2001; 124: 688689.

Samuel S. Becker, M.D.

Vanderbilt University Medical Center

Department of Otolaryngology \& Head and Neck Surgery 7209 Medical Center East, South Tower

Nashville, TN 37232-8605

USA

Tel: +1-609-495-5566

Fax: +1-856-779-0069

E-mail: sam.s.becker@gmail.com 